\title{
Successive approximation method for solving (1+1)-dimensional dispersive long wave equations
}

\author{
Saad A. Manaa*, Fadhil H. Easif, Bewar Y. Ali \\ Department of Mathematics, Faculty of Science, University of Zakho, Kurdistan Region, Iraq
}

\section{ART ICLE INFO}

\section{Article history:}

Received 30 March 2017

Received in revised form

8 July 2017

Accepted 17 July 2017

\section{Keywords:}

Successive approximation method Adomain decomposition method

$(1+1)$-dimensional dispersive long wave equations

\section{Introduction}

The celebrated (1+1)-dimensional dispersive long wave equations (Ablowitz and Clarkson, 1991; Broer, 1975)

$$
\begin{aligned}
& u_{t}+v_{x}+\frac{1}{2}\left(u^{2}\right)_{x}=0, \\
& v_{t}+\left(u v+u+u_{x x}\right)_{x}=0 .
\end{aligned}
$$

Play important roles in nonlinear physics, which describe the evolution of horizontal velocity component $u(x, t)$ of water waves of height $v(x, t)$ propagating in both directions in an infinite narrow channel of finite constant depth. Many properties of (1) have been reported (Broer, 1975; Kaup, 1975; Kupershmidt, 1985).

It is interesting to study the extensions of (1) in higher-dimensional spaces. To date, there exist two prototypical extensions of (1) to cover the situation of wide channel or open seas. Boiti et al. (1987) presented the following (2+1)-dimensional extension related to (1)

$u_{y t}+v_{x x}+\frac{1}{2}\left(u^{2}\right)_{x y}=0$,

$v_{t}+\left(u v+u+u_{x y}\right)_{x}=0$,

in the one-dimensional reduction $u=u(x+y, t)$, $v=v(x+y, t)$, system (2) reduces to system (1) that describes the travel of the shallow water wave.

\footnotetext{
* Corresponding Author.

Email Address: abdnawras@gmail.com (S. A. Manaa) https://doi.org/10.21833/ijaas.2017.08.014

2313-626X/C) 2017 The Authors. Published by IASE. This is an open access article under the CC BY-NC-ND license (http://creativecommons.org/licenses/by-nc-nd/4.0/]
}

Eckhaus (1985) presented another different twodimensional extension of (1)

$u_{t}+v_{x}+\frac{1}{2}\left(u^{2}\right)_{x}=0$

$v_{t x}+\left(u v+u+u_{x x}\right)_{x x}+u_{y y}=0$,

which was obtained in the appropriate approximation from the basic equations of hydrodynamics. It is easy to see that if one makes the transformation either $u=u(x+y, t)$ and $v=$ $v(x+y, t)-1$ or $u=u(x+y, t)$ and $v=v(x+$ $y, t)$, then (3) can also reduce to (1). Therefore, it follows that these two systems (2) and (3) can both reduce to the same system (1) under the proper transformations. But as Boiti et al. (1987) pointed out, system (2) is different from system (3).

\section{Solitary wave solution}

The exact solitary wave solution of the $(2+1)$ dimensional dispersive long wave equations (2) are:

$u(x, y, t)=\alpha+\alpha \tanh \left(\frac{\alpha x+\beta y-\alpha^{2} t+\delta}{2}\right)$,
$v(x, y, t)=-1+\frac{\alpha \beta}{2} \operatorname{sech}^{2}\left(\frac{\alpha x+\beta y-\alpha^{2} t+\delta}{2}\right)$

where $\alpha, \beta$ and $\delta$ are arbitrary constants.

In particular, taking $\beta=\alpha$ and setting $x+y=$ $z$ in system (1), we obtain a special solitary wave solution of $(1+1)$-dimensional dispersive long wave equations (1),

$u(z, t)=\alpha+\alpha \tanh \left(\frac{\alpha z-\alpha^{2} t+\delta}{2}\right)$,
$v(z, t)=-1+\frac{\alpha^{2}}{2} \operatorname{sech}^{2}\left(\frac{\alpha z-\alpha^{2} t+\delta}{2}\right)$,

which was obtained by Bai et al. (2006) and Wang et al. (1996). 


\section{Basic idea of successive approximation method (SAM)}

The method of SAM (Jerri, 1999; Saeed, 2006), provides a method that can, in principle, be used to solve any initial value problem

$\frac{\partial u}{\partial t}=f(u, t), \quad u\left(z, t_{0}\right)=u_{0}$.

It starts by observing that any solution to (8) must also be a solution to

$u(z, t)=u_{0}+\int_{t_{0}}^{t} f(u(z, s), s) d s$,

and then iteratively constructs a sequence of solutions that can get closer and closer to the actual (exact) solutions of (9). The SAM is based on the integral equation (9), as follows:

$u_{0}(z, t)=u_{0}$,

$u_{1}(z, t)=u_{0}+\int_{t_{0}}^{t} f\left(u_{0}(z, s), s\right) d s$,

$u_{2}(z, t)=u_{0}+\int_{t_{0}}^{t} f\left(u_{1}(z, s), s\right) d s$,

$u_{3}(z, t)=u_{0}+\int_{t_{0}}^{t} f\left(u_{2}(z, s), s\right) d s$.

This process can be continued to obtain the $n^{\text {th }}$ approximation,

$u_{n}(z, t)=u_{0}+\int_{t_{0}}^{t} f\left(u_{n-1}(z, s), s\right) d s, \quad n=1,2, \ldots$.

Then determine whether $u_{n}(z, t)$ approaches the solution $u(z, t)$ as $n$ increases. This is done by proving the following:

The sequence $\left\{u_{n}(z, t)\right\}$ converges to a limit $u(z, t)$, that is:

$\lim _{n \rightarrow \infty} u_{n}(z, t)=u(z, t), \quad t_{0} \leq z \leq t$.

The limiting function $u(z, t)$ is a solution of (9) on the interval $t_{0} \leq z \leq t$. The solution $u(z, t)$ of (9) is unique. A proof of these results can be constructed along the lines of the corresponding proof for ordinary differential equations (Coddington, 1995).

\section{SAM applied to $(1+1)$-dimensional dispersive long wave equations}

In this section, we solve the $(1+1)$-dimensional dispersive long wave equations:

$$
\left.\begin{array}{c}
\frac{\partial u(z, s)}{\partial s}=-\frac{\partial v(z, s)}{\partial z}-\frac{1}{2} \frac{\partial u^{2}(z, t)}{\partial z}, \\
\frac{\partial v(z, s)}{\partial s}=-\frac{\partial}{\partial z}(u(z, s)+u(z, s) v(z, s))-\frac{\partial^{3} u(z, s)}{\partial z^{3}},
\end{array}\right\}
$$

with initial solutions

$u(z, 0)=u_{0}$ and $v(z, 0)=v_{0}$,

by using SAM as follows:

Integrating both sides of equation (10) with respect to $s$, from 0 to $t$, we get

$$
\begin{gathered}
u(z, t)=u(z, 0)-\int_{0}^{t}\left(\frac{\partial v(z, s)}{\partial z}+\frac{1}{2} \frac{\partial u^{2}(z, s)}{\partial z}\right) d s \\
v(z, t)=v(z, 0)-\int_{0}^{t}\left(\begin{array}{c}
\frac{\partial}{\partial z}(u(z, s)+u(z, s) v(z, s)) \\
+\frac{\partial^{3} u(z, s)}{\partial z^{3}}
\end{array}\right) d s
\end{gathered}
$$

Using the initial conditions in (11) we get:

$$
\begin{gathered}
u(z, t)=u_{0}-\int_{0}^{t}\left(\frac{\partial v(z, s)}{\partial z}+\frac{1}{2} \frac{\partial u^{2}(z, s)}{\partial z}\right) d s \\
v(z, t)=v_{0}-\int_{0}^{t}\left(\begin{array}{c}
\frac{\partial}{\partial z}(u(z, s)+u(z, s) v(z, s)) \\
+\frac{\partial^{3} u(z, s)}{\partial z^{3}}
\end{array}\right) d s .
\end{gathered}
$$

Start with substituting initial approximation $u_{0}(z, t)$ and $v_{0}(z, t)$ in the integral equation (12) to obtain a first approximation $u_{1}(z, t)$ and $v_{1}(z, t)$

$$
\begin{gathered}
u_{1}(z, t)=u_{0}-\int_{0}^{t}\left(\frac{\partial v_{0}(z, s)}{\partial z}+\frac{1}{2} \frac{\partial u_{0}^{2}(z, s)}{\partial z}\right) d s \\
v_{1}(z, t)=v_{0}-\int_{0}^{t}\left(\begin{array}{c}
\frac{\partial}{\partial z}\left(u_{0}(z, s)+u_{0}(z, s) v_{0}(z, s)\right) \\
+\frac{\partial^{3} u_{0}(z, s)}{\partial z^{3}}
\end{array}\right) d s .
\end{gathered}
$$

Then this $u_{1}(z, t)$ and $v_{1}(z, t)$ is substituted again in the integral of (12) after replacing $t$ by $s$ to obtain a second approximation $u_{2}(z, t)$ and $v_{2}(z, t)$,

$$
\begin{aligned}
& u_{2}(z, t)=u_{0}-\int_{0}^{t}\left(\frac{\partial v_{1}(z, s)}{\partial z}+\frac{1}{2} \frac{\partial u_{1}^{2}(z, s)}{\partial z}\right) d s, \\
& v_{2}(z, t)=v_{0}-\int_{0}^{t}\left(\begin{array}{c}
\frac{\partial}{\partial z}\left(u_{1}(z, s)+u_{1}(z, s) v_{1}(z, s)\right) \\
+\frac{\partial^{3} u_{1}(z, s)}{\partial z^{3}}
\end{array}\right) d s .
\end{aligned}
$$

This process can be continued to obtain the $n^{\text {th }}$ approximation

$$
\begin{aligned}
& u_{n}(z, t)=u_{0}-\int_{0}^{t}\left(\frac{\partial v_{n-1}(z, s)}{\partial z}+\frac{1}{2} \frac{\partial u_{n-1}^{2}(z, s)}{\partial z}\right) d s, \\
& v_{n}(z, t)=v_{0}-\int_{0}^{t}\left(\begin{array}{c}
\frac{\partial}{\partial z}\left(\begin{array}{c}
u_{n-1}(z, s) \\
+u_{n-1}(z, s) v_{n-1}(z, s)
\end{array}\right) \\
+\frac{\partial^{3} u_{n-1}(z, s)}{\partial z^{3}}
\end{array}\right) d s,
\end{aligned}
$$

For $n=1,2, \ldots$. To solve the equation (14), we use the initial approximation $u_{0}(z, t)$ and $v_{0}(z, t)$ which are given in equation (6) and (7), respectively. Using the iteration formula (14), we can obtain $u_{1}(z, t), u_{2}(z, t), u_{3}(z, t), v_{1}(z, t), v_{2}(z, t)$ and $v_{3}(z, t)$ as follows:

$$
\begin{aligned}
& u_{1}(z, t)=\alpha+\alpha \tanh \left(\frac{\alpha z+\delta}{2}\right)-\frac{\alpha^{3}}{2} \operatorname{sech}^{2}\left(\frac{\alpha z+\delta}{2}\right) t \\
& u_{2}(z, t)=\alpha+\alpha \tanh \left(\frac{\alpha z+\delta}{2}\right)-\frac{\alpha^{3}}{2} \operatorname{sech}^{2}\left(\frac{\alpha z+\delta}{2}\right) t- \\
& \frac{\alpha^{5}}{4} \operatorname{sech}^{2}\left(\frac{\alpha z+\delta}{2}\right) \tanh \left(\frac{\alpha z+\delta}{2}\right) t^{2}+ \\
& \frac{\alpha^{7}}{12} \operatorname{sech}^{4}\left(\frac{\alpha z+\delta}{2}\right) \tanh \left(\frac{\alpha z+\delta}{2}\right) t^{3}, \\
& u_{3}(z, t)=\alpha+\alpha \tanh \left(\frac{\alpha z+\delta}{2}\right)-\frac{\alpha^{3}}{2} \operatorname{sech}^{2}\left(\frac{\alpha z+\delta}{2}\right) t- \\
& \frac{\alpha^{5}}{4} \operatorname{sech}^{2}\left(\frac{\alpha z+\delta}{2}\right) \tanh \left(\frac{\alpha z+\delta}{2}\right) t^{2}+\frac{\alpha^{7}}{24} \operatorname{sech}^{2}\left(\frac{\alpha z+\delta}{2}\right)(1- \\
& \left.3 \tanh ^{2}\left(\frac{\alpha z+\delta}{2}\right)\right) t^{3}-\frac{5 \alpha^{9}}{192} \operatorname{sech}^{4}\left(\frac{\alpha z+\delta}{2}\right)(1- \\
& \left.2 \tanh ^{2}\left(\frac{\alpha z+\delta}{2}\right)-5 \tanh ^{2}\left(\frac{\alpha z+\delta}{2}\right)+\frac{5}{18} \tanh ^{3}\left(\frac{\alpha z+\delta}{2}\right)\right) t^{4}+
\end{aligned}
$$




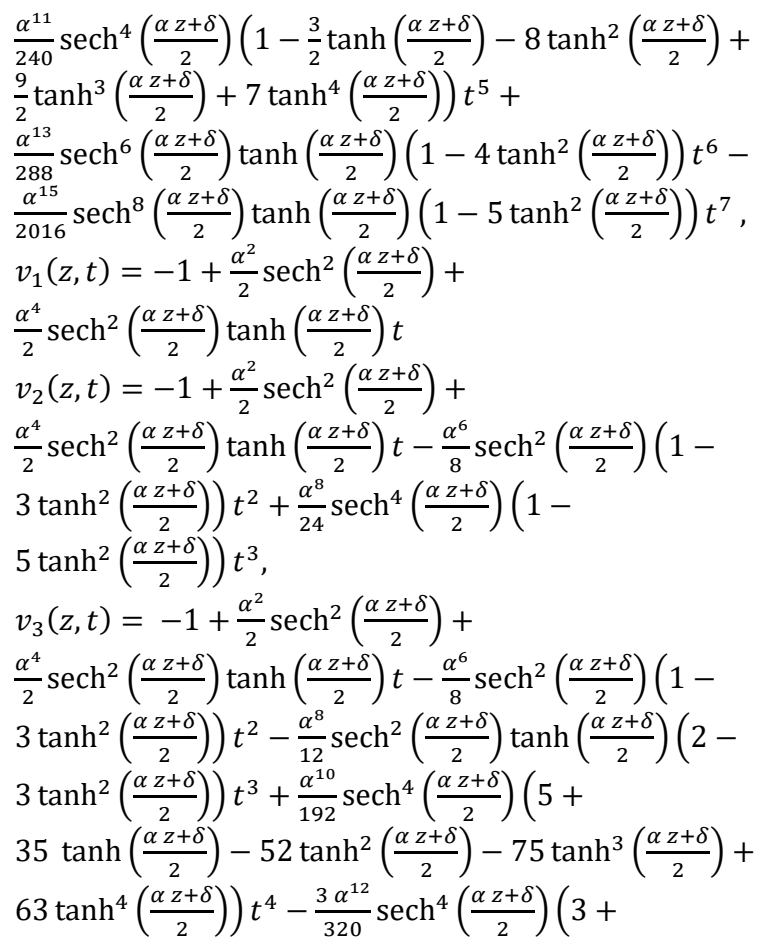

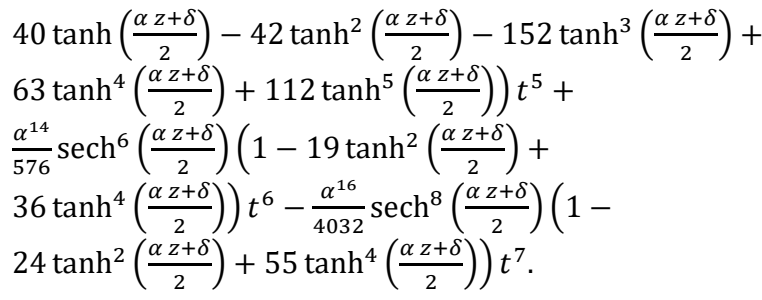

\section{Results and discussion}

We solved the $(1+1)$-dimensional dispersive long wave equations by SAM. Compared between 3rdorder approximate solutions of SAM and ADM (Mohamed, 2010) with exact solitary solutions. The results it shown by data and surfaces see in Tables $1 \mathrm{a}, 1 \mathrm{~b}, 2 \mathrm{a}$, and $2 \mathrm{~b}$ and Figs. 1, 2, and 3. And we found the effects for $\alpha$ and $\delta$ on the solution of $(1+1)$ dimensional dispersive long wave equations as shown in Figs. 4a, 4b, 5a, and 5b. It shown that $\alpha$ has effects on long and short in wave and $\delta$ has effects on move wave only.

Table 1a: Exact solution, $3^{\text {rd }}$-order approximation solutions ADM (Mohamed, 2010) and SAM of $u(z, t)$ when $\alpha=2, \delta=0$ and $z=-0.01$

\begin{tabular}{cccc}
\hline$t$ & Exact & ADM & SAM \\
\hline 0 & 1.98000066664 & 1.98000066664 & 1.98000066664 \\
0.01 & 1.9400179935224 & 1.9400179965071 & 1.9400178614665 \\
0.02 & 1.9000832500842 & 1.9000833114428 & 1.9000811646242 \\
0.03 & 1.8602282193671 & 1.860228598649 & 1.8602178003544 \\
0.04 & 1.8204844305057 & 1.8204858453275 & 1.8204519384 \\
0.05 & 1.7808830595711 & 1.7808870386801 & 1.7808047973543 \\
0.06 & 1.7414548327879 & 1.7414641659086 & 1.7412947478293 \\
0.07 & 1.7022299327534 & 1.7022492142149 & 1.7019374154519 \\
0.08 & 1.6632379082584 & 1.6632741708006 & 1.662745783697 \\
0.09 & 1.6245075882634 & 1.6245710228678 & 1.6237302965644 \\
0.1 & 1.5860670005411 & 1.5861717576181 & 1.584898961109 \\
\hline
\end{tabular}

Table 1b: Exact solution, $3^{\text {rd }}$-order approximation solutions ADM (Mohamed, 2010) and SAM of $v(z, t)$ when $=2, \delta=0$ and

\begin{tabular}{cccc}
\multicolumn{4}{c}{$z=-0.01$} \\
\hline $\mathrm{t}$ & Exact & ADM & SAM \\
\hline 0 & 0.99980001333258 & 0.99980001333258 & 0.99980001333258 \\
0.01 & 0.99820107944946 & 0.99820086649064 & 0.99820111308927 \\
0.02 & 0.99500832154314 & 0.99500491883283 & 0.99500884803683 \\
0.03 & 0.99023192466936 & 0.9902147296339 & 0.99023454277574 \\
0.04 & 0.98388708015456 & 0.98383285816862 & 0.98389524331417 \\
0.05 & 0.97599388320855 & 0.97586186371178 & 0.97601363254549 \\
0.06 & 0.96657719825563 & 0.96630430553815 & 0.96661796371 \\
0.07 & 0.95566649352597 & 0.95516274292249 & 0.95574201102401 \\
0.08 & 0.9432956467829 & 0.94243973513958 & 0.94342503665934 \\
0.09 & 0.92950272436413 & 0.9281378414642 & 0.92971177325638 \\
0.1 & 0.91432973597948 & 0.9122596211711 & 0.91465242115369 \\
\hline
\end{tabular}

Table 2a: Absolute error between exact solution and $3^{\text {rd }}$ order approximation solutions ADM (Mohamed, 2010) and

\begin{tabular}{ccc} 
SAM of $u(z, t)$ & when $\alpha=2, \delta=0$ and $z=-0.0$ \\
\hline $\mathrm{t}$ & $\mid$ Exact - ADM $\mid$ & Exact - SAM | \\
\hline 0 & 0 & 0 \\
0.01 & $2.9847 \mathrm{E}-09$ & $1.32056 \mathrm{E}-07$ \\
0.02 & $6.13586 \mathrm{E}-08$ & $2.08546 \mathrm{E}-06$ \\
0.03 & $3.79282 \mathrm{E}-07$ & $1.0419 \mathrm{E}-05$ \\
0.04 & $1.41482 \mathrm{E}-06$ & $3.24921 \mathrm{E}-05$ \\
0.05 & $3.97911 \mathrm{E}-06$ & $7.82622 \mathrm{E}-05$ \\
0.06 & $9.33312 \mathrm{E}-06$ & 0.000160085 \\
0.07 & $1.92815 \mathrm{E}-05$ & 0.000292517 \\
0.08 & $3.62625 \mathrm{E}-05$ & 0.000492125 \\
0.09 & $6.34346 \mathrm{E}-05$ & 0.000777292 \\
0.1 & 0.000104757 & 0.001168039 \\
Total Error & 0.000238906 & 0.003013449 \\
\hline
\end{tabular}

Table 2b: Absolute error between exact solution and $3^{\text {rd }}$ order approximation solutions ADM (Mohamed, 2010) and

\begin{tabular}{ccc} 
SAM of $v(z, t)$ & when $\alpha=2, \delta=0$ and $z=-0.01$ \\
\hline $\mathrm{t}$ & $\mid$ Exact - ADM $\mid$ & $\mid$ Exact -SAM $\mid$ \\
\hline 0 & 0 & 0 \\
0.01 & $2.12959 \mathrm{E}-07$ & $3.36398 \mathrm{E}-08$ \\
0.02 & $3.40271 \mathrm{E}-06$ & $5.26494 \mathrm{E}-07$ \\
0.03 & $1.7195 \mathrm{E}-05$ & $2.61811 \mathrm{E}-06$ \\
0.04 & $5.4222 \mathrm{E}-05$ & $8.16316 \mathrm{E}-06$ \\
0.05 & 0.000132019 & $1.97493 \mathrm{E}-05$ \\
0.06 & 0.000272893 & $4.07655 \mathrm{E}-05$ \\
0.07 & 0.000503751 & $7.55175 \mathrm{E}-05$ \\
0.08 & 0.000855912 & 0.00012939 \\
0.09 & 0.001364883 & 0.000209049 \\
0.1 & 0.002070115 & 0.000322685 \\
Total Error & 0.005274605 & 0.000808498 \\
\hline
\end{tabular}




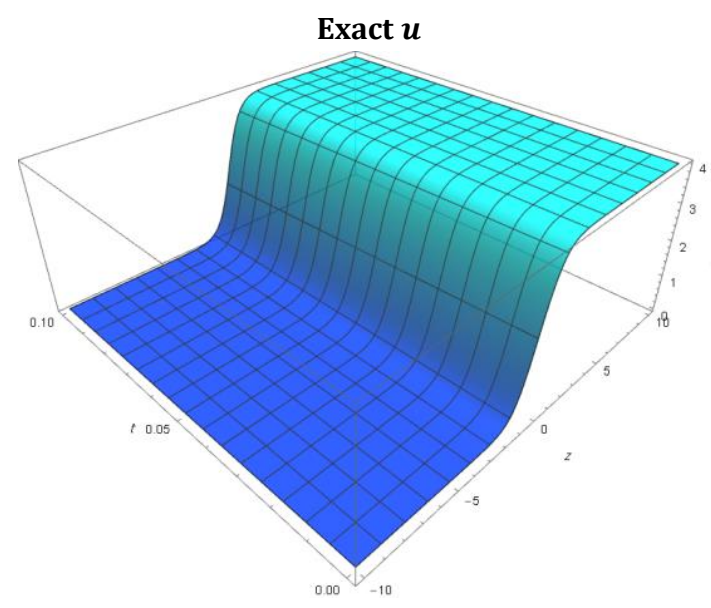

(a)

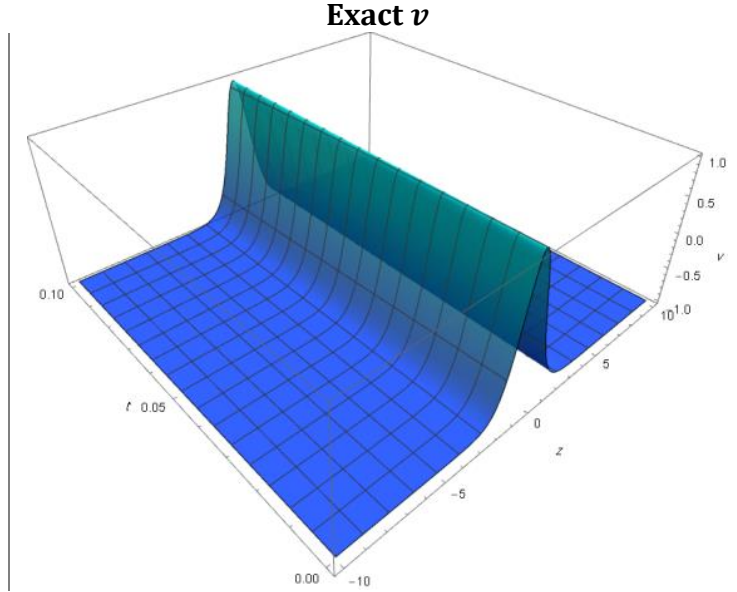

(b)

Fig. 1: The surfaces of exact solutions for $u(z, t)$ and $v(z, t)$, when $\alpha=2, \delta=0$ and $z \in[-10,10]$ at $0 \leq t \leq 0.1$

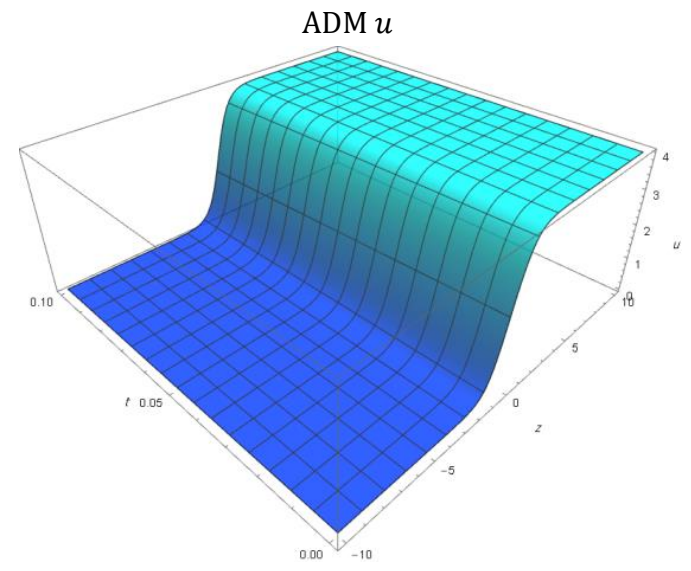

(a)

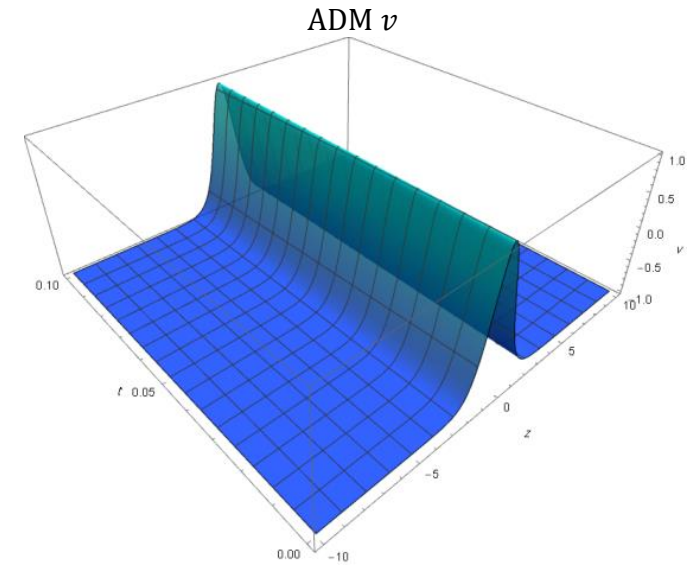

(b)

Fig. 2: The surfaces of ADM solutions for $u(z, t)$ and $v(z, t)$, when $\alpha=2, \delta=0$ and $z \in[-10,10]$ at $0 \leq t \leq 0.1$

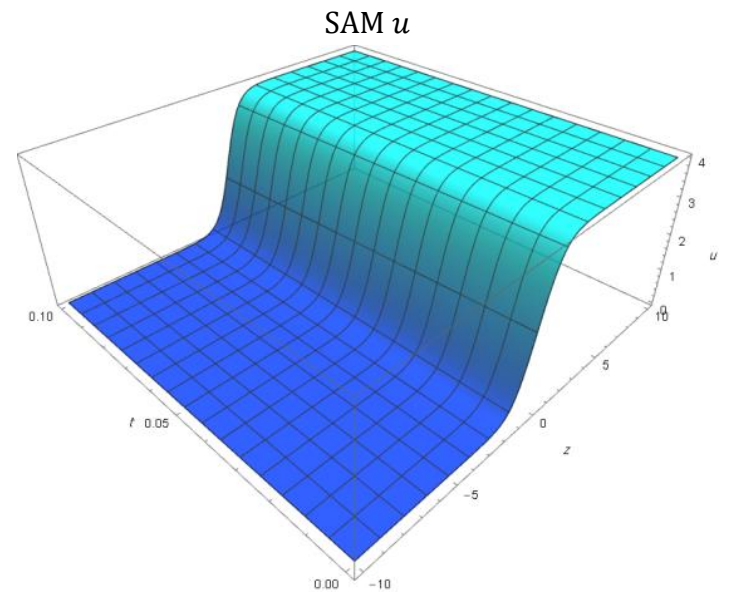

(a)

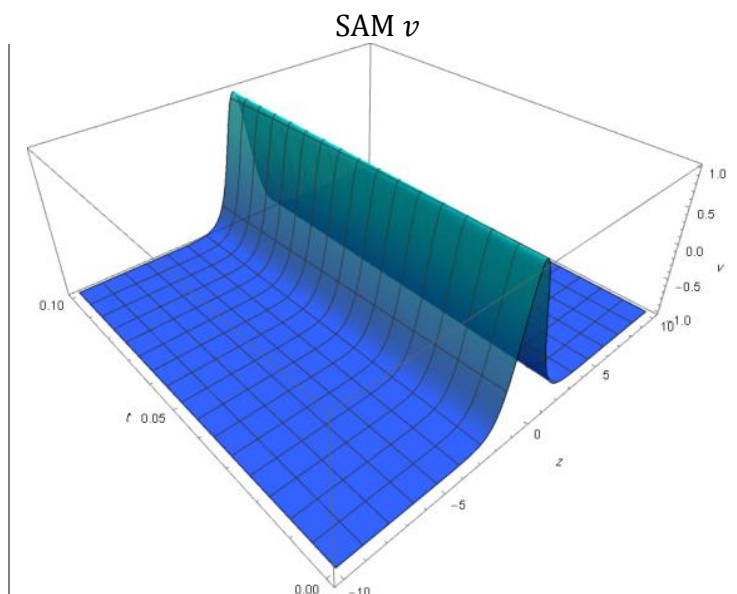

(b)

Fig. 3: The surfaces of SAM solutions for $u(z, t)$ and $v(z, t)$, when $\alpha=2, \delta=0$ and $z \in[-10,10]$ at $0 \leq t \leq 0.1$ Effects of $\alpha$ in $u(z, t)$

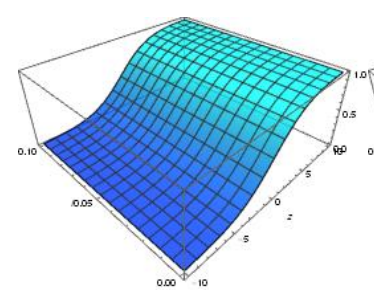

$\alpha=0.5$

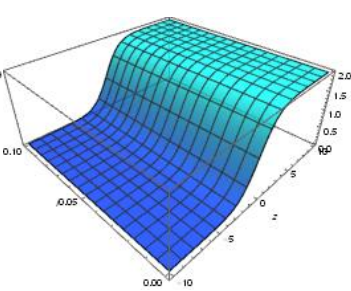

$\alpha=1$

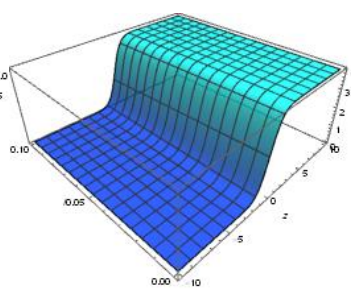

$\alpha=2$

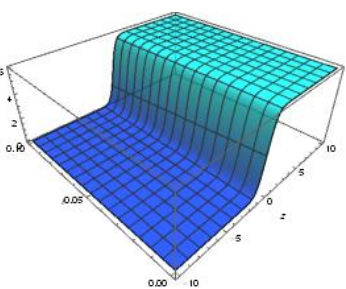

$\alpha=3$

Fig. 4a: The surfaces of exact solution of $u(z, t)$, when $\alpha=0.5,1,2,3$ at $\delta=0, z \in[-10,10], 0 \leq t \leq 0.1$ 


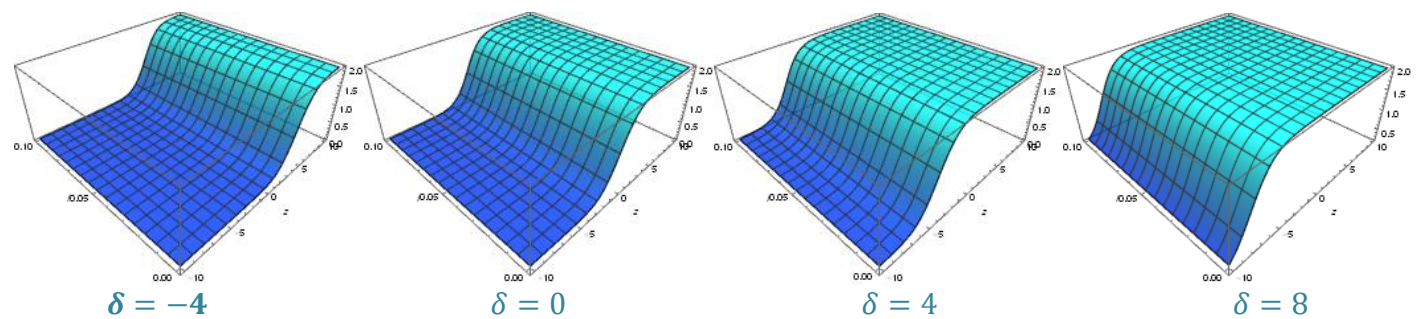

Fig. 4b: The surfaces of exact solution of $u(z, t)$, when $\delta=-4,0,4,8$ at $\alpha=1, z \in[-10,10], 0 \leq t \leq 0.1$

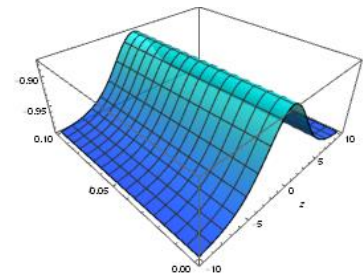

$\alpha=0.5$

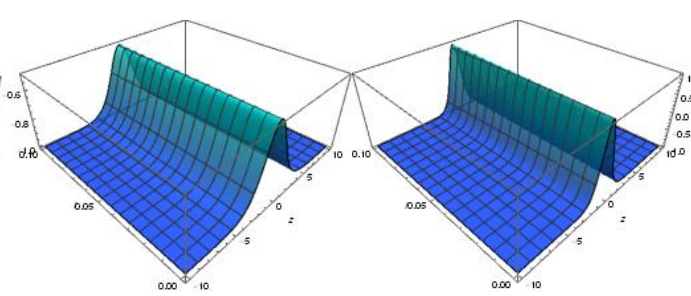

$\alpha=2$

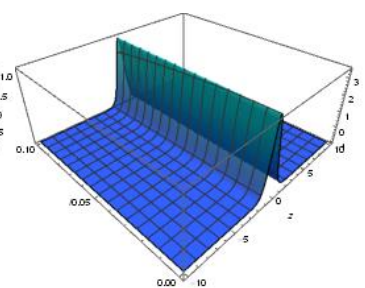

$\alpha=3$

Fig. 5a: The surfaces of exact solution of $v(z, t)$, when $\alpha=0.5,1,2,3$ at $\delta=0, z \in[-10,10], 0 \leq t \leq 0.1$

\section{Effects of $\delta$ in $v(z, t)$}
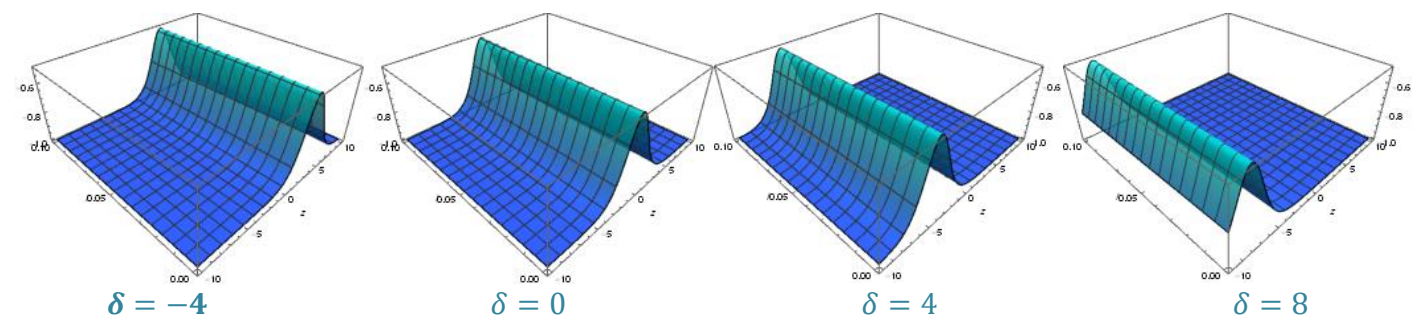

Fig. 5b: The surfaces of exact solution of $v(z, t)$, when $\delta=-4,0,4,8$ at $\alpha=1, z \in[-10,10], 0 \leq t \leq 0.1$

\section{Conclusion}

In this paper, the SAM successfully applied to obtain approximate solutions of the $(1+1)$ dimensional dispersive long wave. We showed that successive approximation method easier, faster and more accurate than Adomain's decomposition method as shown especially in Tables $1 \mathrm{~b}$ and $2 \mathrm{~b}$ and Figs. $2 \mathrm{~b}$ and $3 \mathrm{~b}$. Finally, it has become clear that $\alpha$ and $\delta$ have effects on solutions.

\section{Acknowledgment}

We thank the editor and the referee for their comments.

\section{List of symbols}

\section{SAM}

ADM

Greek symbols

$\alpha, \beta$ and $\delta$
Successive Approximate Method Adomain Decomposition Method

Constants

\section{References}

Ablowitz MJ and Clarkson PA (1991). Solitons, nonlinear evolution equations and inverse scattering. Cambridge University Press, Cambridge, UK.

Bai CL, Zhao H, and Han JG (2006). Application of an extended homogeneous balance method to new exact solutions of nonlinear evolution equations. Czechoslovak Journal of Physics, 56(3): 237-242.

Boiti M, Leon JJP, and Pempinelli F (1987). Spectral transform for a two spatial dimension extension of the dispersive long wave equation. Inverse Problems, 3(3): 371-387.

Broer LJF (1975). Approximate equations for long water waves. Applied Scientific Research, 31(5): 377-395.

Coddington EA (1995). An introduction to ordinary differential equations. Prentice Hall of India, New Delhi, India.

Eckhaus W (1985). Preprint 404. University of Utrecht, Utrecht, Netherlands.

Jerri A (1999). Introduction to integral equations with applications. John Wiley and Sons, Hoboken, USA.

Kaup DJ (1975). A higher-order water-wave equation and the method for solving it. Progress of Theoretical Physics, 54(2): 396-408. 
Kupershmidt BA (1985). Mathematics of dispersive water waves. Communications in Mathematical Physics, 99(1): 51-73.

Mohamed MA (2010). Comparison differential transformation technique with adomian decomposition method for dispersive long-wave equations in $(2+1)$-dimensions. Applications and Applied Mathematics: An International Journal (AAM), 5(1): 148-166.
Saeed RK (2006). Computational methods for solving system of linear volterra integral and integro-differential equations. Ph.D. Dissertation, University of Salahaddin/Erbil-Collage of Science, Erbil, Iraqi Kurdistan.

Wang M, Zhou Y, and Li Z (1996). Application of a homogeneous balance method to exact solutions of nonlinear equations in mathematical physics. Physics Letters A, 216(1-5): 67-75. 